\title{
On-pump Cardiac Surgery Enhances Platelet Renewal and Impairs Aspirin Pharmacodynamics: Effects of Improved Dosing Regimens
}

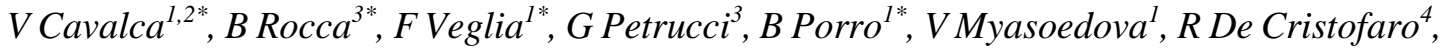

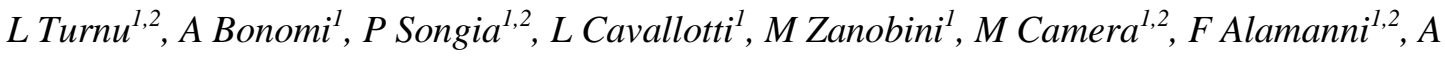 \\ Parolari $^{5}$, C Patrono ${ }^{3}$, E Tremoli ${ }^{1}$
}

${ }^{1}$ Monzino Cardiology Center, IRCCS, Milan, Italy; ${ }^{2}$ University of Milan, Milan, Italy; Departments of ${ }^{3}$ Pharmacology, and ${ }^{4}$ Hematology, Catholic University, Rome, Italy; ${ }^{5}$ Policlinico San Donato Hospital, IRCCS, San Donato Milanese, Italy.

*Drs. Cavalca, Rocca and Veglia equally contributed to the study

Running Title: Aspirin pharmacodynamics after cardiac surgery

Address for correspondence:

Bianca Rocca, MD, PhD

Department of Pharmacology

Catholic University School of Medicine

Largo F. Vito 1

00168 Rome, Italy

Phone: +390630154253

Email: b.rocca@tiscali.it; bianca.rocca@unicatt.it;

Word count: 4124

References: 50

Figures: 5

Tables: 2

Keywords: aspirin, pharmacodynamics, thromboxane, prostacyclin, cyclooxygenase-1. 


\begin{abstract}
On-pump cardiac surgery may trigger inflammation and accelerate platelet
\end{abstract} cyclooxygenase-1 renewal, thereby modifying low-dose aspirin pharmacodynamics.

Thirty-seven patients on standard aspirin $100 \mathrm{mg}$ once-daily were studied before surgery and randomized within 36 hours post-surgery to $100 \mathrm{mg}$ once-daily, $100 \mathrm{mg}$ twice-daily or $200 \mathrm{mg}$ once-daily for 90 days. On day 7 post-surgery, immature and mature platelets, platelet mass, thrombopoieitin, glycocalicin, leukocytes, C-reactive protein, and interleukin-6 significantly increased. Interleukin-6 significantly correlated with immature platelets. At day 7, patients randomized to $100 \mathrm{mg}$ once-daily showed a significant increase in serum thromboxane $(\mathrm{TX}) \mathrm{B}_{2}$ within the 24-hour dosing interval and urinary $\mathrm{TXA}_{2}$ metabolite (TXM) excretion. Aspirin $100 \mathrm{mg}$ twice-daily lowered serum $\mathrm{TXB}_{2}$ and prevented post-surgery TXM increase $(\mathrm{p}<0.01)$, without affecting prostacyclin metabolite excretion.

After cardiac surgery, shortening the dosing interval, but not doubling the once-daily dose, rescues the impaired antiplatelet effect of low-dose aspirin and prevents platelet activation associated with acute inflammation and enhanced platelet turnover. 
Surgical treatment of patients with coronary artery disease (CAD), including coronary artery bypass grafting $(\mathrm{CABG})$, or aortic valve stenosis of atherosclerotic origin represents an important therapeutic approach. However, the success of the intervention depends not only on the surgical procedure and peri-operative management, but also on an adequate post-operative antithrombotic therapy. Antiplatelet treatment before and immediately after cardiac surgery influences the clinical outcomes, including graft patency after $\mathrm{CABG},(1,2)$ consistent with the evidence that platelet activation plays an important role in the pathophysiology of vascular complications.(3) Aspirin (100 to $325 \mathrm{mg}$ once daily [od]) is currently recommended before and early after cardiac surgery, $(1,4)$ unless the bleeding risk outweighs the thrombotic risk.(1) However, in spite of antiplatelet prophylaxis, the incidence of major vascular events is quite high in the first month following $\mathrm{CABG}$ (up to $17 \%$ in a recent trial),(5) and only slightly decreases between 2 and 12 months. $(1,6,7)$

Several lines of evidence question the efficacy of a standard low-dose aspirin od regimen used in cardiac surgery. Some studies reported an 'aspirin resistant' or 'hyperreactive' platelet phenotype immediately after cardiac surgery, $(8,9)$ as well as an impaired capacity of low-dose aspirin to fully inhibit platelet cyclooxygenase (COX)-1-derived thromboxane $(\mathrm{TX}) \mathrm{A}_{2} .(10,11) \mathrm{We}$ and others have reported higher-than-expected residual platelet function, a lower degree of platelet inhibition(8, 11-13) and/or enhanced expression of the COX-2 isoform in circulating platelets, immediately after CABG or aortic valve replacement performed with on-pump cardiopulmonary bypass (CPB).(10) Moreover, the clinical efficacy of low-dose aspirin after cardiac surgery has been questioned by a meta-analysis of placebo-controlled studies, which indirectly compared low (75-150 mg) vs. medium (300-325 mg) aspirin doses.(14) Within the limitations of an indirect comparison, the relative risk reduction of graft occlusion was $26 \%$ and $45 \%$, with low- and medium-dose regimens as compared to placebo, respectively. We have 
previously shown that aspirin $325 \mathrm{mg}$ od achieves greater platelet $\mathrm{TXA}_{2}$ inhibition than $100 \mathrm{mg}$ od post-CABG.(12)

Different mechanisms have been proposed to explain enhanced platelet activation and impaired inhibition early after on-pump cardiac surgery. The on-pump CPB procedure can activate platelets(15) and trigger inflammation.(16,17) A higher fraction of peripheral platelets expressing COX-2 has been detected 5 days after surgery.(10) Because COX-2 is only expressed in newly-released platelets, (18) this finding may reflect an increased platelet turnover after on-pump surgery.(10) Accelerated platelet renewal associated with type 2 diabetes mellitus or essential thrombocythemia has been shown to require more frequent aspirin dosing to achieve complete and persistent platelet COX-1 inhibition.(1921) Moreover, aspirin $81 \mathrm{mg}$ aspirin four-times daily (qid) was shown to be superior vs. the standard $81 \mathrm{mg}$ od regimen in inhibiting platelet $\mathrm{TXA}_{2}$ post-CPB.(22)

In the present study, we investigated whether the standard od regimen of low-dose $(100 \mathrm{mg})$ aspirin has a differential inhibitory effect on platelet COX-1-dependent $\mathrm{TXA}_{2}$ production before and after CPB surgery for CABG and/or aortic-valve replacement, and whether impaired inhibition can be rescued by shortening the dosing interval or doubling the od dose, early after (7 days) and 90 days post-surgery. We measured different inflammatory indexes, and several parameters related to platelet turnover to characterize the determinants of impaired pharmacodynamics of low-dose aspirin across surgery. We also evaluated the effects of different aspirin regimens on the in vivo biosynthesis of vascular prostacyclin $\left(\mathrm{PGI}_{2}\right)$, an important endogenous mechanism of endothelial thromboresistance.(3)

\section{RESULTS}

\section{Study patients}

The design of the study is shown in Figure 1. Among 44 patients initially recruited, 7 were 
found non-compliant with aspirin during the run-in phase and excluded from final analyses (see Methods for non-compliance assessment). The main characteristics of the remaining 37 patients overall as well as according to post-surgery randomization arm are reported in Supplementary Table 1. No statistically significant differences among the randomized groups were observed. Moreover, patients with isolated aortic valve stenosis $(n=10)$ did not differ from patients with CAD undergoing elective CABG $(n=24)$ for different pre-operative parameters including activated partial thromboplastin time (aPTT), platelet count, serum thromboxane B2 (sTXB2), high-sensitivity C-reactive protein (hs-CRP) and urinary $\mathrm{PGI}_{2}$ metabolite (PGIM) excretion to any statistically significant extent (23) (Supplementary Table 2).

Urinary excretion of thromboxane A2 metabolite (TXM) (24) and PGIM, as well as platelet-related variables measured before surgery at 8 am (T1) and 8 pm (T2), showed no major circadian differences (Table 1).

The most relevant intra- and early post-operative surgical characteristics in the entire population and according to the randomized treatment are reported in Supplementary Table 3. No differences among groups were observed for intra-operative aortic clamp, CPB and intubation time. Moreover, the amount of blood loss in the first 48 hours after surgery measured in the drainages, did not differ to any statistically significant extent between patients with previous isolated aortic valve stenosis and patients who underwent CABG (556 [386-726] and 677 [567-786] mL, respectively, $\mathrm{p}=0.23$ ). No major hemorrhagic or thrombotic complications, and/or re-intervention occurred during and after hospital discharge, until the end of study (day 90 post-randomization) (Supplementary Table 3).

\section{Effects of cardiac surgery on platelet renewal and inflammatory indexes}

The values of thrombopoietic and inflammatory cytokines, and different hematopoietic indexes measured at T1, T3 and T5 (Figure 1) are reported in Table 2. As compared to pre-surgery (T1), immature and mature platelet counts, platelet mass, 
thrombopoietin, interleukin (IL)-6, hs-CRP, glycocalicin, glycocalicin index (GCI), total leukocyte, neutrophil, monocyte and eosinophil counts, all significantly increased at day 7 post-surgery (T3) (Table 2 and Supplementary Figures 1, 2, 3). IL-6 showed the largest post-surgery increase as compared to other soluble indexes (Figure 2A). When expressed as fold-change vs. pre-surgery values, IL-6 increases were significantly correlated with hsCRP ( $\mathrm{r}=0.41, \mathrm{p}=0.013)$ and thrombopoietin $(\mathrm{r}=0.33, \mathrm{p}=0.046)$ fold-increases. Moreover, post-operative IL-6 significantly correlated with immature platelet counts (Figure 2B). The post-surgical increases in total and immature platelet counts, platelet mass, IL-6, thrombopoietin, and glycocalicin were consistent with an ongoing condition of enhanced platelet regeneration, while the increase in GCI likely reflected a recent platelet destruction $(25,26)$ during $\mathrm{CPB}$. Consistent with these findings, a significant inverse correlation between percent immature platelet fraction (IPF) and total platelet count was observed both at 8 am and $8 \mathrm{pm}$ on day 7 post-surgery, which was not observed in the same patients before surgery (Supplementary Figure 4) and 90 days after surgery (Supplementary Figure 5). In addition, at day 90 , most of the platelet regeneration and inflammatory indexes returned to pre-surgery values (Table 2, Supplementary Figures 1, 2, 3, 5).

\section{Impaired aspirin pharmacodynamics of the standard aspirin regimen after cardiac} surgery

We assessed whether cardiac surgery and $\mathrm{CPB}$ affect the pharmacodynamics of the standard aspirin od regimen and the level of in vivo platelet activation. Twenty-four to 36 hours after surgery, at $8 \mathrm{pm}$, patients were randomized to start one of three open-label regimens: $100 \mathrm{mg}$ od (8 pm), $100 \mathrm{mg}$ twice daily (bid) $(8 \mathrm{am}, 8 \mathrm{pm})$ or $200 \mathrm{mg}$ od (8 pm). Patients were sampled at 8 am (T3) and 8 pm (T4) on day 7 and at 8 am (T5) on day 90 postsurgery (Figure 1). We measured $\mathrm{sTXB}_{2}$ as a surrogate biomarker of aspirin efficacy, because it specifically reflects the antiplatelet pharmacodynamics of aspirin (27), in

accordance

with

European

regulatory

guidelines 
(http://www.ema.europa.eu/docs/en_GB/document_library/Scientific_guideline/2009/09/ WC500003340.pdf).

In patients randomized to continue aspirin $100 \mathrm{mg}$ od post-surgery, the peak value of $\mathrm{STXB}_{2}$ measured in the 24-hour posological interval, significantly increased from 2.2 [1-4.2] pre-surgery to 3.4 [1.9-10.9] $\mathrm{ng} / \mathrm{mL}$ post-surgery (medians and interquartile range [IQR]; $\mathrm{p}=0.04)$. Moreover, $\mathrm{sTXB}_{2}$ values significantly increased between 8 am and $8 \mathrm{pm}(12$ and 24 hours after dosing, respectively) on day 7 post-surgery (Figure 3), as reflected by the slope of the $\mathrm{sTXB}_{2}$ recovery rate (Figure 4).

Consistent with inadequate platelet inhibition by the standard aspirin regimen post-surgery, urinary TXM excretion at the end of the dosing interval on day 7 post-surgery $(8 \mathrm{pm}, \mathrm{T} 4)$ was significantly higher than the corresponding pre-surgery value (T2) (Supplementary Figure 6A). At variance with $\mathrm{sTXB}_{2}$ or urinary TXM, platelet aggregation in whole blood as assessed by the VerifyNow ${ }^{\circledR}$ Aspirin assay on day 7 post-surgery did not display any statistically significant variation at 8 pm vs. 8 am (Supplementary Table 4).

At 90 days post-surgery, $\mathrm{sTXB}_{2}$ values in this group of patients remained numerically higher than the corresponding pre-surgery values $(\mathrm{p}=0.06)$, and was not significantly different from the 8 am values on day 7, while urinary TXM excretion did not differ from pre-surgery values to any statistically significant extent (data not shown).

\section{Improved postsurgery aspirin pharmacodynamics with a different dosing regimen}

We investigated whether post-surgery aspirin pharmacodynamics could be improved by a more frequent dosing or doubling the od dose.

Patients randomized to $200 \mathrm{mg}$ od had similar 24-hour $\mathrm{STXB}_{2}$ peak values before and after surgery: $3.4[2-4.4] \mathrm{ng} / \mathrm{ml}$ vs. 2.9 [1.5-5.2] $\mathrm{ng} / \mathrm{mL}$, respectively $(\mathrm{p}=0.19)$. Moreover, $\mathrm{sTXB}_{2}$ significantly increased between 8 am and 8 pm on day 7 post- surgery (Figure 3), with a 12 - to 24 -hour recovery slope similar to pre-surgery values. 
Urinary TXM excretion at 8 pm of day 7 post-surgery (T4) was significantly higher than the corresponding pre-surgery value (Supplementary Figure 6A). At 90 days (T5), sTXB 2 and urinary TXM values returned to pre-surgery levels in this treatment group (data not shown). Platelet aggregation showed no statistically significant changes at any time point (Supplementary Table 4).

At variance with the other groups, patients randomized to aspirin $100 \mathrm{mg}$ bid showed a $30 \%$ lower 24-hour $\mathrm{sTXB}_{2}$ peak value at day 7 after vs. before surgery: 2.7 [24.4] $\mathrm{ng} / \mathrm{ml}$ vs. 3.8 [2.5-6.5] $\mathrm{ng} / \mathrm{mL}$, respectively $(\mathrm{p}=0.03)$. Moreover, $\mathrm{sTXB}_{2}$ values were similar at 8 am and $8 \mathrm{pm}$, with no measurable recovery of platelet COX-1 activity (Figure 3). Furthermore, $\mathrm{sTXB}_{2}$ values at $8 \mathrm{pm}$ on day 7 (T4) were significantly lower than the corresponding pre-surgery values $(\mathrm{T} 2)(\mathrm{p}=0.03)$. Consistent with complete and persistent platelet COX-1 suppression, urinary TXM excretion was not increased at $8 \mathrm{pm}$ on day 7 post-surgery (Supplementary Figure 6A). Platelet aggregation did not show any statistically significant changes at 8 am before-surgery, 7 days and 3 months post-surgery (Supplementary Table 4). At 90 days after surgery, sTXB 2 and urinary TXM levels were comparable to baseline (data not shown).

We also compared the $\mathrm{sTXB}_{2}$ recovery slopes measured between 12 and 24 hours after dosing on day 7 post-surgery in the 3 randomized groups, as an index of the rate of recovery of platelet COX-1 activity during the dosing interval. $(19,20)$ The $\mathrm{sTXB}_{2}$ recovery slopes before surgery were not significantly different among the three treatment groups (data not shown). However, the post-operative slope was significantly lower in the $100 \mathrm{mg}$ bid group as compared to both $100 \mathrm{mg}$ od and $200 \mathrm{mg}$ od groups (Figure 4). The sTXB 2 recovery slopes on day 7 were not significantly different between the 100 and $200 \mathrm{mg}$ od groups (Figure 4). At day 90 after surgery, $\mathrm{sTXB}_{2}$ levels at $8 \mathrm{am}$ were higher in the $100 \mathrm{mg}$ od group as compared to the other two treatments (Supplementary Figure 7). 


\section{Effects of different aspirin regimens on vascular PGI $_{2}$ biosynthesis}

No major circadian variations in urinary PGIM excretion were detected on day 7 post-surgery (Table 1). As compared to pre-surgery, PGIM excretion significantly increased at day 7 after surgery (Supplementary Figure 6B and Table 1) and this increase was observed within each treatment group, independently of the aspirin regimen. The urinary PGIM fold-change on day 7 post- vs. pre-surgery correlated with IL-6 ( $\mathrm{r}=0.42$, $\mathrm{p}=0.01)$ and hs-CRP $(\mathrm{r}=0.40, \mathrm{p}=0.02)$ fold-changes.

At day 90, PGIM excretion returned to baseline values in the $100 \mathrm{mg}$ bid and 200 $\mathrm{mg}$ od groups, while in the $100 \mathrm{mg}$ od group, urinary PGIM excretion remained significantly higher than pre-surgery $(\mathrm{p}<0.05$, data not shown).

\section{DISCUSSION}

Early thrombosis is a major cause of CABG graft occlusion, and $\sim 50 \%$ of major thrombotic complications occur within the first month after cardiac surgery, in spite of low-dose aspirin prophylaxis started early after the intervention. $(6,7)$

Our study examined platelet kinetics and aspirin pharmacodynamics pre- and postcardiac surgery, to address four related questions: 1) whether the extent and duration of platelet cyclooxygenase (COX)-1 inhibition by the standard aspirin regimen is impaired after surgery coincident with enhanced platelet activation in vivo; 2) whether indexes of recent platelet destruction and active regeneration in the week after surgery are suggestive of accelerated platelet renewal; 3) whether a shorter dosing interval and/or a higher dose of aspirin rescues aspirin altered pharmacodynamics; 4) whether the vascular production of the antithrombotic $\mathrm{PGI}_{2}$ is increased by cardiac surgery and reduced by more intensive aspirin regimens. To our knowledge, this is the first study where these mechanistic and clinical questions were simultaneously asked with the appropriate analytical tools, in patients undergoing $\mathrm{CBP}$ for $\mathrm{CABG}$ or aortic valve replacement. 
We repeatedly measured $\mathrm{STXB}_{2}$, a highly-specific index of platelet COX-1 activity, $(27,28)$ to assess its 24 -hour peak value and 12 - to 24 -hour recovery rate after a witnessed aspirin intake, to definitively exclude the confounding effect of non-compliance. The standard aspirin regimen (100 $\mathrm{mg}$ od) became significantly less effective in the first week after vs. before surgery. Moreover, the faster kinetics of $\mathrm{sTXB}_{2}$ recovery during the 24hour dosing interval was associated with significantly increased urinary TXM excretion. While $\mathrm{sTXB}_{2}$ reflects the maximal biosynthetic capacity of platelet COX-1 triggered by endogenous thrombin ex vivo, $(27,28)$ urinary TXM excretion reflects the actual rate of TXA $_{2}$-dependent platelet activation in vivo.(24) Thus, our findings demonstrate that incomplete and shorter-lasting inhibition of platelet COX-1 activity by a standard low-dose aspirin regimen is unable to suppress enhanced platelet activation in vivo associated with cardiac surgery and CPB. Given the strikingly non-linear relationship between inhibition of platelet COX-1 activity and of platelet activation in vivo,(29) it is not surprising that incomplete suppression of the former is associated with inadequate inhibition of the latter. The largely negative findings of platelet aggregation measurements ex vivo are consistent with the major limitations and high variability of platelet function assays reported by pharmacologic studies (29) as well as by the logarithmic, rather than linear relationship observed between the Verify-Now and $\operatorname{sTXB}_{2 .}$ (20) Moreover, recent randomized trials failed to demonstrate the clinical utility of these assay in adjusting antiplatelet therapy to improve its efficacy and safety. $(30,31)$

Impaired aspirin pharmacodynamics early after cardiac surgery was associated with significant increases in immature platelets, total platelets, platelet mass, thrombopoieitin, IL-6, glycocalicin, leukocytes, and hs-CRP. These data are consistent with previous findings separately showing increased interleukins, including IL-6 $(16,32)$ and immature $(10)$ or total platelets $(16,22,32)$ post-CABG and/or CPB. Moreover, the statistically significant inverse correlation between the IPF and platelet count observed on 
day 7 post-surgery is consistent with an enhanced thrombopoiesis, and highly reminiscent of other conditions of platelet destruction and regeneration. $(25,26,33)$ Importantly, IL-6 at day 7 post-surgery showed the largest increase (Figure 2A) and was highly correlated with immature platelet counts (Figure 2B). IL-6 can control inflammation through hsCRP(34) and is also involved in modulating megakaryocyte differentiation and acute megakaryocyte fragmentation with immediate platelet release associated with inflammatory states. $(35,36)$ IL-6 mediates thrombocytosis, which characterizes inflammatory bowel disorders in humans and animal models by increasing peripheral immature platelets with enhanced reactivity. $(36,37)$ These findings closely mirror the post-operative pattern observed in our patients. The acute changes in thrombopoietic indexes in our patients were largely reversible to a pre-surgery pattern by 90 days postsurgery, consistently with previous data (16). Thus, our findings suggest that the month(s) immediately following cardiac surgery represent a potential clinical setting where to test the cardiovascular effects of newer anti-inflammatory strategies.(34)

Under conditions of faster platelet renewal driven by platelet destruction and IL-6 increase, a complete and persistent 24-hour inhibition of platelet COX-1 activity is expected to be achieved more effectively by shortening the aspirin dosing interval (from 24 to 12 hours) rather than by increasing the od dose, as shown in other disorders. $(19,20)$ Our results are consistent with this theoretical expectation, by showing that a $100 \mathrm{mg}$ bid regimen is superior to od regimens of either 100 or $200 \mathrm{mg}$, in terms of lower sTXB 2 peak value and recovery rate (Figure 3), with complete suppression of in vivo platelet activation associated with cardiac surgery (Supplementary Figure 6A). A higher suppression of in vivo platelet activation, as reflected by urinary TXM excretion, by a bid vs. od regimen of lowdose aspirin administration was previously reported in ET patients.(20) This pharmacodynamic superiority of a bid regimen persisted throughout 3 months postsurgery. The same approach was used by Ivert et al., in a recently published study of 
aspirin dosing (75 mg od or bid vs. $160 \mathrm{mg}$ od) after CABG surgery.(38) They also found greater efficacy of aspirin $75 \mathrm{mg}$ bid vs. od but not a clear difference vs. $160 \mathrm{mg}$ od with regard to $\mathrm{sTXB}_{2}, 1$ and 3 months after CABG. A qid regimen of aspirin $81 \mathrm{mg}$ was previously shown to be more effective than $81 \mathrm{mg}$ od in suppressing sTXB 2 within 7 days post-CABG.(22) However, a qid regimen may substantially reduce compliance, its vascular safety in terms of $\mathrm{PGI}_{2}$ suppression is unknown, and data beyond day 7 were not reported.(22) The same study also showed that aspirin $325 \mathrm{mg}$ od was more effective than $81 \mathrm{mg}$ od,(22) consistent with our previous comparison of $325 \mathrm{vs} .100 \mathrm{mg}$ od for 5 days post-CABG.(12) However, aspirin $325 \mathrm{mg}$ od has been shown to inhibit vascular $\mathrm{PGI}_{2}$ by at least 50\%.(39) This degree of inhibition may adversely impact cardiovascular safety, as suggested by the increased risk of major vascular events following a two-week course of COX-2 inhibition post-CABG. $(40,41)$ Hence, we investigated the effect of different aspirin regimens on the $\mathrm{COX}$-2-dependent in vivo biosynthesis of the antithrombotic prostanoid $\mathrm{PGI}_{2} .(3,42)$ Its major urinary metabolite, PGIM, increased on day 7 postsurgery, which may have reflected endothelial damage caused by cardiac surgery and/or a homeostatic response to enhanced platelet activation. Neither the $100 \mathrm{mg}$ bid nor the 200 mg od aspirin regimen blunted the PGIM increase, suggesting a negligible effect on vascular COX-2 activity, consistent with our previous finding in essential thrombocythemia.(43)

Our study has some limitations. First of all, it included two different groups of patients, i.e. those with $\mathrm{CAD}$ undergoing $\mathrm{CABG}$ and a smaller group of aortic valve stenosis undergoing valve replacement. It has been reported that aortic valve stenosis can be associated with acquired type $2 \mathrm{~A}$ von Willebrand defect,(44) due to abnormal hemodynamics in the stenotic region,(45) resulting in platelet function abnormalities and gastrointestinal bleeding (Heyde's syndrome).(46) While we can exclude the presence of a symptomatic Heyde's syndrome in our aspirin-treated patients based on the exclusion 
criteria (major bleeding in the previous 6 months), a minor asymptomatic von Willebrand defect cannot be excluded in the absence of von Willebrand multimer determination.(44) However, a clinically meaningful von Willebrand defect can be reasonably excluded in our patients given the absence of aPTT prolongation in the aortic valve stenosis group, and the similar amounts of blood loss immediately after surgery between patients with and without aortic valve stenosis. Moreover, all patients underwent the same procedure associated with $\mathrm{CPB}$, with similar intra-operative parameters in all the randomized arms and no differences in bleeding loss in the 48 hours after surgery. An acquired type $2 \mathrm{~A}$ von Willebrand defect would have increased post-surgical bleeding. In addition, because of its scope and size, our study was not designed to detect differences in clinical outcomes associated with different aspirin regimens. Therefore, whether and to what extent an improved aspirin regimen may provide a therapeutic advance, remains unknown. However, the size of the unmet therapeutic need for antithrombotic prophylaxis during and after cardiac surgery (5) is sufficiently large to warrant further clinical investigation addressing the optimal antiplatelet therapy in this setting.

In conclusion, shortening the dosing interval, but not doubling the dose, rescues the impaired antiplatelet effect of low-dose aspirin without affecting the in vivo biosynthesis of the antithrombotic $\mathrm{PGI}_{2}$, and prevents platelet activation associated with acute inflammation and enhanced platelet turnover immediately after cardiac surgery. (Figure 5). The clinical translation of these findings will need to be assessed by adequately sized randomized trials of improved vs conventional antiplatelet regimens.

\section{METHODS}

\section{Patients and design of the study}

We performed a randomized, controlled, intervention study between January 2012 and June 2013, in patients undergoing elective CABG and/or aortic valve replacement using bioprosthesis, for calcific, non-rheumatic stenosis, according to routine on-pump 
CPB and standard surgical procedures.(47) The study was approved by the institutional Ethics Committee (EudraCT2011-001488-40) and conducted according to the Declaration of Helsinki. All patients signed an informed consent.

Inclusion criteria were: age between 55 and 80 years, ejection fraction $>30 \%$, sinus rhythm, ongoing treatment with enteric-coated aspirin $100 \mathrm{mg}$ od(Cardioaspirin ${ }^{\circledR}$, Bayer, Italy). Exclusion criteria were: myocardial infarction, ischemic stroke or major bleeding within the previous 6 months, severe renal or liver disease, hypertension or hypercholesterolemia that were not pharmacologically controlled, current cigarette smoking, pregnancy or lactation, aspirin intolerance, known primary or secondary bleeding or hematological disorders, platelet count $<150 \times 10^{3} / \mu \mathrm{L}$ or $>450 \times 10^{3} / \mu \mathrm{L}$, use of antiplatelet agents other than low-dose aspirin, anticoagulants and/or non-steroidal anti-inflammatory drugs (NSAIDs). In addition, routine coagulation tests (prothrombin time, aPTT, fibrinogen) were performed before surgery, in order to exclude subclinical acquired or congenital coagulation deficits. If any of the coagulation tests was outside the normal range interval, patients were excluded from being considered for this study. No additional antithrombotic drugs, except for heparin, were administered during hospitalization or follow-up. Only paracetamol or tramadol were allowed for post-operative pain.

The study design is depicted in Figure 1. During the 7-10 days before hospitalization (run-in phase), eligible patients were asked to take their daily aspirin tablet in the evening (from 8 to $10 \mathrm{pm}$ ). The evening of hospitalization, patients underwent blood withdrawal to check for compliance (T0) and took a witnessed 100-mg aspirin tablet. The following day, patients were sampled at 8 am (T1) and 8 pm (T2) (i.e. 12 and 24 hours after the witnessed aspirin intake), then aspirin was withdrawn 12-20 hours before surgery. Twenty-four to 36 hours after surgery, at $8 \mathrm{pm}$ patients were randomized to start one of three open-label regimens: $100 \mathrm{mg}$ od $(8 \mathrm{pm}), 100 \mathrm{mg}$ bid $(8 \mathrm{am}, 8 \mathrm{pm})$ or $200 \mathrm{mg}$ od (8 pm). Patients were sampled at 8 am (T3) and $8 \mathrm{pm}$ (T4) on day 7 post-surgery. After 
hospital discharge patients continued their randomized treatment for 90 days, and they were sampled again at 8 am of day 90 ( \pm 1 day) (T5, end of study) and resumed their standard aspirin thereafter (Figure 1).

\section{Biochemical and hematological measurements}

At T1 (pre-surgery), T3 (day 7 post-surgery) and T5 (day 90 post-surgery) we assessed: routine blood counts, routine chemistry, hs-CRP, IL-6, thrombopoietin, glycocalicin by commercial assays (see Supplementary Methods). We also assessed the

IPF (Sysmex XE 2100, Sysmex Co., Kobe, Japan), and platelet function in whole blood by the VerifyNow-Aspirin assay (VerifyNow ${ }^{\circledR}$ Aspirin, Accumetrix, San Diego, CA). The absolute count of immature platelets was calculated as IPF $x$ platelet count $\left(\mathrm{x} 10^{3} / \mu \mathrm{L}\right) / 100$. Platelet mass was calculated as mean platelet volume $(M P V) \times$ platelet count $\left(\mathrm{x} 10^{3} / \mu \mathrm{L}\right)$; the glycocalicin index (GCI) was calculated as [glycocalicin-ng/ml] $\times 250,000 /$ platelet count $\left(x 10^{3} / \mu L\right)$, according to standard equations. $(25,26)$

\section{Blood and urine prostanoid metabolite measurements}

Platelet COX-1 activity was assessed at each time point (from T0 to T5) by measuring the $\mathrm{TXB}_{2}$ in serum $\left(\mathrm{sTXB}_{2}\right)$ after whole blood clotting at $37^{\circ} \mathrm{C}$ for 1 hour, as previously described.(28) Serum $\mathrm{TXB}_{2}$ measurements performed 12 and 24 hours after aspirin intake allow investigating the recovery of platelet COX-1 activity during the 24hour dosing interval. $(19,48)$ In particular, the 12 - to 24 -hour recovery rate of $\mathrm{sTXB}_{2}$ was calculated as the slope of the linear fitting of these experimental time points and expressed as $\mathrm{ng} / \mathrm{ml} /$ hour.(19)

The systemic biosynthesis of $\mathrm{TXA}_{2}$, a non-invasive index of in vivo platelet activation, was assessed at each time point by measuring its major urinary metabolite, i.e., 11-dehydro-TXB 2 (TXM).(24) The major urinary $\mathrm{PGI}_{2}$ metabolite, i.e., 2,3-dinor-6-keto- 
$\mathrm{PGF}_{1 \alpha}(\mathrm{PGIM})$, was measured to investigate the systemic biosynthesis of vascular $\mathrm{PGI}_{2}$ in vivo.(23) Both urinary TXM and PGIM were measured by liquid chromatography-tandem mass spectrometry as previously described, $(43,49)$ and expressed as $\mathrm{pg} / \mathrm{mg}$ of creatinine.

\section{Assessment of compliance}

Compliance was assessed by pill counting and patient's interview at hospital entry However, given the well known limitations of these indirect methods for checking drug adherence,(50) we also used a biochemical method by comparing $\mathrm{sTXB}_{2}$ levels at $8 \mathrm{pm}$ on the evening of hospitalization (T0) vs. the levels at 24 hours following a witnessed administration of aspirin (T2). The $\mathrm{sTXB}_{2}$ levels at $\mathrm{T} 0$ were interpreted to reflect patient's compliance during the previous 3-4 days. If the absolute values of $\mathrm{sTXB}_{2}$ at $\mathrm{T} 0$ and $\mathrm{T} 2$ differed by $>20 \%$, the patient was further investigated about drug intake, timing of aspirin intake and NSAID use at home and considered non-compliant, as previously described.(48) The cut-off of $20 \%$ takes into account the variability of the $\mathrm{sTXB}_{2}$ assay, i.e. $\sim 10 \%$, as well as the evidence that aspirin poor responsiveness, or 'resistance', is a stable phenotype over repeated $\mathrm{sTXB}_{2}$ measurements. (20)

\section{Statistical analyses}

To test the ability of the standard aspirin regimen $\left(100 \mathrm{mg}\right.$ od) to inhibit platelet $\mathrm{TXA}_{2}$ biosynthesis steadily during the 24-hour dosing interval, we considered as primary endpoint the highest $\mathrm{sTXB}_{2}$ concentration over 24 hours, detected either at 8 am or $8 \mathrm{pm}$ (which we will define as 'peak' $\mathrm{sTXB}_{2}$ ). To evaluate the impact of cardiac surgery on the pharmacodynamic efficacy of the standard aspirin regimen, the peak $\mathrm{sTXB}_{2}$ value was considered in the $100 \mathrm{mg}$ od group before and after surgery. At least 11 patients were required to detect as statistically significant, with a 2-tailed alpha of 0.05 and $80 \%$ power, an absolute change of $\mathrm{sTXB}_{2}$ peak value $\geq 2 \mathrm{ng} / \mathrm{ml}$ between post- and pre-surgery, assuming 
a baseline $\mathrm{sTXB}_{2}$ peak level of $4.9 \pm 2 \mathrm{ng} / \mathrm{mL}$ (mean $\pm \mathrm{SD}$ ).(29) Moreover, the same sample size provided $80 \%$ power to detect a difference $\geq 3 \mathrm{ng} / \mathrm{mL}$ in the $\mathrm{sTXB}_{2}$ peak value between aspirin $100 \mathrm{mg}$ od and $100 \mathrm{mg}$ bid or $200 \mathrm{mg}$ od comparing the three different groups after surgery. Therefore, assuming a 20\% drop-out, we aimed to recruit at least 41 patients, to have at least 33 evaluable patients. We chose $\mathrm{sTXB}_{2}$ as a surrogate biomarker of aspirin efficacy, in accordance with european regulatory guidelines (http://www.ema.europa.eu/docs/en_GB/document_library/Scientific_guideline/2009/09/ WC500003340.pdf).

Numerical variables were expressed as mean and standard deviation (SD), median and inter-quartile range (IQR), frequency or percentage, as appropriate. Kuskal-Wallis and Wilcoxon rank-sum tests were employed for between-group comparisons; Wilcoxon signed-rank test for within-group comparisons; Spearman's rank correlation for associations. Tests were two-sided. P-values $<0.05$ were considered statistically significant. Analyses were performed by SAS version 9.4 (SAS Institute Inc., Cary, NC). 


\section{Study Highlights}

\section{What is the current knowledge on the topic?}

On-pump cardiac surgery is used for revascularization of patients with coronary artery and atherosclerotic valve disease. The incidence of major vascular events remains quite high in the first month after surgery, in spite of the currently recommended low-dose aspirin regimen.

\section{What question this study addresses?}

This study addressed the question as to whether cardiac surgery impairs aspirin pharmacodynamics through increased platelet turnover, and evaluated the antiplatelet efficacy and vascular effects of improved aspirin regimens.

\section{What this study adds to our knowledge}

Shortening the dosing interval, but not doubling the once-daily dose, rescues the impaired antiplatelet pharmacodynamics of low-dose aspirin without affecting the in vivo biosynthesis of vascular prostacyclin, and prevents in vivo platelet activation associated with acute inflammation and enhanced platelet turnover following on-pump cardiac surgery.

\section{How this might change clinical pharmacology or translational science}

Effective antithrombotic prophylaxis after on-pump cardiac surgery for atherosclerotic diseases remains an unmet therapeutic need. Our proof-of-concept study demonstrates that a twice daily low-dose aspirin regimen can improve drug responsiveness and prevent in vivo platelet activation The clinical translation of these findings will need to be assessed by adequately sized randomized trials of improved vs conventional antiplatelet regimens. 


\title{
Acknowledgements
}

This work was supported by grants from: the Italian Ministry of Health 'Ricerca Corrente' to Centro Cardiologico Monzino; 'Università Cattolica, Linea D1-2015' to BR; and 'Università Cattolica, Linea D1-2014' to RDC.

\section{Conflict of interest}

Dr. Carlo Patrono has received consulting and lecture fees from Amgen, AstraZeneca and Bayer AG, and an institutional grant from Bayer AG for investigatorinitiated research on platelet activation and inhibition in diabetes mellitus; he is a member of the Scientific Advisory Board of the International Aspirin Foundation. The other Authors have nothing to disclose.

\author{
Author's Contribution \\ $\mathrm{CP}, \mathrm{ET}, \mathrm{BR}$ designed research \\ VC, GP, BP, PS, LC, MZ, VM, MC, AB, FA, LT, AP performed research \\ FV, BR and RDC analyzed data. \\ $\mathrm{CP}, \mathrm{ET}, \mathrm{BR}, \mathrm{VC}, \mathrm{FV}$ wrote the manuscript
}

\section{References}

(1) Sousa-Uva, M. et al. Expert position paper on the management of antiplatelet therapy in patients undergoing coronary artery bypass graft surgery. Eur Heart J 35, 1510-4 (2014).

(2) Zimmermann, N., Gams, E. \& Hohlfeld, T. Aspirin in coronary artery bypass surgery: new aspects of and alternatives for an old antithrombotic agent. Eur $J$ Cardiothorac Surg 34, 93-108 (2008).

(3) Davi, G. \& Patrono, C. Platelet activation and atherothrombosis. $N$ Engl J Med 357, 2482-94 (2007).

(4) Hillis, L.D. et al. 2011 ACCF/AHA guideline for coronary artery bypass graft surgery: executive summary: a report of the American College of Cardiology Foundation/American Heart Association Task Force on Practice Guidelines. J Thorac Cardiovasc Surg 143, 4-34 (2012). 
(5) Myles, P.S. et al. Stopping vs. Continuing Aspirin before Coronary Artery Surgery. N Engl J Med 374, 728-37 (2016).

(6) Serruys, P.W. et al. Percutaneous coronary intervention versus coronaryartery bypass grafting for severe coronary artery disease. $N$ Engl $\mathrm{J} \mathrm{Med} \mathrm{360,}$ 961-72 (2009).

(7) Hakeem, A., Garg, N., Bhatti, S., Rajpurohit, N., Ahmed, Z. \& Uretsky, B.F. Effectiveness of percutaneous coronary intervention with drug-eluting stents compared with bypass surgery in diabetics with multivessel coronary disease: comprehensive systematic review and meta-analysis of randomized clinical data. J Am Heart Assoc 2, eooo354 (2013).

(8) Ferraris, V.A. \& Bolanos, M.D. Use of antiplatelet drugs after cardiac operations. Semin Thorac Cardiovasc Surg 26, 223-30 (2014).

(9) Storey, R.F. Exploring mechanisms of graft occlusion toward improved outcomes in coronary artery bypass graft surgery. J Am Coll Cardiol 57, 107880 (2011).

(10) Zimmermann, N. et al. Functional and biochemical evaluation of platelet aspirin resistance after coronary artery bypass surgery. Circulation 108, 5427 (2003).

(11) Bednar, F., Osmancik, P., Hlavicka, J., Jedlickova, V., Paluch, Z. \& Vanek, T. Aspirin is insufficient in inhibition of platelet aggregation and thromboxane formation early after coronary artery bypass surgery. $J$ Thromb Thrombolysis 27, 394-9 (2009).

(12) Brambilla, M. et al. Effect of two doses of aspirin on thromboxane biosynthesis and platelet function in patients undergoing coronary surgery. Thromb Haemost 103, 516-24 (2010).

(13) Zimmermann, N. et al. Platelet inhibition by aspirin after aortic valve replacement. J Thorac Cardiovasc Surg 131, 1392-3 (2006).

(14) Lim, E. et al. Indirect comparison meta-analysis of aspirin therapy after coronary surgery. Bmj 327, 1309 (2003).

(15) Sniecinski, R.M. \& Chandler, W.L. Activation of the hemostatic system during cardiopulmonary bypass. Anesth Analg 113, 1319-33 (2011).

(16) Li, N., Astudillo, R., Ivert, T. \& Hjemdahl, P. Biphasic pro-thrombotic and inflammatory responses after coronary artery bypass surgery. $J$ Thromb Haemost 1, 470-6 (2003).

(17) Parolari, A. et al. Biomarkers in Coronary Artery Bypass Surgery: Ready for Prime Time and Outcome Prediction? Front Cardiovasc Med 2, 39(2016).

(18) Rocca, B. et al. Cyclooxygenase-2 expression is induced during human megakaryopoiesis and characterizes newly formed platelets. Proc Natl Acad Sci U S A 99, 7634-9 (2002).

(19) Rocca, B. et al. The recovery of platelet cyclooxygenase activity explains interindividual variability in responsiveness to low-dose aspirin in patients with and without diabetes. J Thromb Haemost 10, 1220-30 (2012).

(20) Pascale, S. et al. Aspirin-insensitive thromboxane biosynthesis in essential thrombocythemia is explained by accelerated renewal of the drug target. Blood 119, 3595-603 (2010).

(21) Spectre, G., Arnetz, L., Ostenson, C.G., Brismar, K., Li, N.\& Hjemdahl, P. Twice daily dosing of aspirin improves platelet inhibition in whole blood in patients with type 2 diabetes mellitus and micro- or macrovascular complications. Thromb Haemost 106, 491-9 (2009). 
(22) Paikin, J.S. et al. Multiple daily doses of acetyl-salicylic acid (ASA) overcome reduced platelet response to once-daily ASA after coronary artery bypass graft surgery: a pilot randomized controlled trial. J Thromb Haemost 13, 448-56 (2015).

(23) FitzGerald, G.A., Brash, A.R., Falardeau, P.\& Oates, J.A. Estimated rate of prostacyclin secretion into the circulation of normal man. J Clin Invest $\mathbf{6 8}$, 1272-6 (1981).

(24) Ciabattoni, G., Pugliese, F., Davi, G., Pierucci, A., Simonetti, B.M.\& Patrono, C. Fractional conversion of thromboxane B2 to urinary 11-dehydrothromboxane B2 in man. Biochim Biophys Acta 992, 66-70 (1989).

(25) Kurata, Y. et al. Diagnostic value of tests for reticulated platelets, plasma glycocalicin, and thrombopoietin levels for discriminating between hyperdestructive and hypoplastic thrombocytopenia. Am J Clin Pathol 115, 656-64 (2001).

(26) Strom, T.S. Numerical analysis of in vivo platelet consumption data from ITP patients. BMC Hematol 15, 14 (2015).

(27) Patrono, C. et al. Clinical pharmacology of platelet cyclooxygenase inhibition. Circulation 72, 1177-84 (1985).

(28) Patrono, C. et al. Low dose aspirin and inhibition of thromboxane B2 production in healthy subjects. Thromb Res 17, 317-27 (1980).

(29) Santilli, F. et al. Platelet cyclooxygenase inhibition by low-dose aspirin is not reflected consistently by platelet function assays: implications for aspirin "resistance". J Am Coll Cardiol 53, 667-77 (2009).

(30) Collet, J.P. et al. Bedside monitoring to adjust antiplatelet therapy for coronary stenting. N Engl J Med 367, 2100-9 (2012).

(31) Cayla, G. et al. Platelet function monitoring to adjust antiplatelet therapy in elderly patients stented for an acute coronary syndrome (ANTARCTIC): an open-label, blinded-endpoint, randomised controlled superiority trial. Lancet, (2016).

(32) Arazi, H.C. et al. Impaired anti-platelet effect of aspirin, inflammation and platelet turnover in cardiac surgery. Interact Cardiovasc Thorac Surg 10, 863- 7 (2009).

（33） Louwes, H., De Wolf, J.T., Houwerzijl, E. \&Vellenga, E. Evaluation assays measuring platelet kinetics in bone marrow and peripheral blood. An overview. Nucl Med Commun 23, 581-90 (2002).

(34) Ridker, P.M. From C-Reactive Protein to Interleukin-6 to Interleukin-1: Moving Upstream To Identify Novel Targets for Atheroprotection. Circ Res 118, 14556 (2016).

(35) Nishimura, S. et al. IL-1 $\alpha$ induces thrombopoiesis through megakaryocyte rupture in response to acute platelet needs. J Cell Biol 11, 453-66. (2015).

(36) Senchenkova, E.Y. et al. Interleukin-6 mediates the platelet abnormalities and thrombogenesis associated with experimental colitis. Am J Pathol 183, 173-81 (2013).

（37） Yan, S.L., Russell, J.\& Granger, D.N. Platelet activation and platelet-leukocyte aggregation elicited in experimental colitis are mediated by interleukin- 6 . Inflamm Bowel Dis 20, 353-62 (2014).

(38) Ivert, T. et al. Platelet function one and three months after coronary bypass surgery in relation to once or twice daily dosing of acetylsalicylic acid. Thromb Res 149, 64-9 (2017). 
(39) FitzGerald, G.A. et al. Endogenous biosynthesis of prostacyclin and thromboxane and platelet function during chronic administration of aspirin in man. J Clin Invest 71, 676-88 (1983).

(40) Nussmeier, N.A. et al. Complications of the COX-2 inhibitors parecoxib and valdecoxib after cardiac surgery. N Engl J Med 352, 1081-91 (2005).

(41) Furberg, C.D., Psaty, B.M.\&FitzGerald, G.A.Parecoxib, valdecoxib, and cardiovascular risk. Circulation 111, 249 (2005).

(42) McAdam, B.F., Catella-Lawson, F., Mardini, I.A., Kapoor, S., Lawson, J.A. \& FitzGerald, G.A. Systemic biosynthesis of prostacyclin by cyclooxygenase (COX)-2: the human pharmacology of a selective inhibitor of COX-2. Proc Natl Acad Sci U S A 96, 272-7 (1999).

(43) Cavalca, V. et al. In vivo prostacyclin biosynthesis and effects of different aspirin regimens in patients with essential thrombocythaemia. Thromb Haemost 112, 118-27 (2015).

(44) Vincentelli, A. et al. Acquired von Willebrand syndrome in aortic stenosis. $N$ Engl J Med 349, 343-9 (2003).

(45) Back, M., Gasser,T.C., Michel,J.B.\&Caligiuri, G. Biomechanical factors in the biology of aortic wall and aortic valve diseases. Cardiovasc Res 99, 232-41 (2013).

(46) Natorska, J., Mazur, P. \& Undas, A. Increased bleeding risk in patients with aortic valvular stenosis: From new mechanisms to new therapies. Thromb Res 139, 85-9 (2016).

(47) Alamanni, F. et al. On- and off-pump coronary surgery and perioperative myocardial infarction: an issue between incomplete and extensive revascularization. Eur J Cardiothorac Surg 34, 118-26 (2008).

(48) Zaccardi, F. et al. In Vivo Platelet Activation and Aspirin Responsiveness in Type 1 Diabetes. Diabetes 65, 503-9 (2016).

(49) Cavalca, V. et al. Simultaneous quantification of 8-iso-prostaglandin-F(2alpha) and 11-dehydro thromboxane $\mathrm{B}(2)$ in human urine by liquid chromatographytandem mass spectrometry. Anal Biochem 397, 168-74 (2010).

(50) Kubica, A., Obonska, K., Fabiszak, T. \& Kubica, J. Adherence to antiplatelet treatment with $\mathrm{P} 2 \mathrm{Y} 12$ receptor inhibitors. Is there anything we can do to improve it? A systematic review of randomized trials. Curr Med Res Opin 32, 1441-51 (2016). 


\section{Figure Legends}

Figure 1. Design of the study. The flow chart depicts the design of the study and timing of blood and urine sampling. The evening of hospitalization, patients underwent blood withdrawal to check for compliance (T0) and took a witnessed 100-mg aspirin tablet. The following day, patients were sampled at 8 am (T1) and 8 pm (T2). Aspirin was withdrawn 12-20 hours before surgery and re-started after surgery when patients were randomized to $100 \mathrm{mg}$ od, $100 \mathrm{mg}$ bid or $200 \mathrm{mg}$ od for 90 days. Patients were sampled at 8 am (T3) and 8 pm (T4) on day 7 and at 8 am of day 90 ( \pm 1 day) (T5, end-of-study) post-surgery. IPF: immature platelet fraction; PGIM: PGI $_{2}$ metabolite; TXM: TXA 2 metabolite; hs-CRP: high sensitivity C-reactive protein

Figure 2. Platelet-related, thrombopoietic and inflammatory indexes on day 7 postsurgery. Panel A: The figure shows the box-whisker plots representing median, IQR and outlier values of fold-increase in glycocalicin index, glycocalicin, thrombopoietin, Creactive protein and interleukin- 6 at 7 day post- vs. pre- surgery; $n=37$. Panel B: The figure depicts the statistically significant correlation between IL-6 levels and immature platelet counts at 8 am on day 7 post-surgery (T3).

Figure 3. Postsurgery serum $\mathrm{TXB}_{2}$ associated with different aspirin regimens. Panel A: Serum $\mathrm{TXB}_{2}$ individual values at 12 and 24 hours ( 8 am and $\left.8 \mathrm{pm}\right)$ associated with different aspirin regimens at day 7 post surgery in patients randomized to $100 \mathrm{mg}$ od, $200 \mathrm{mg}$ od or $100 \mathrm{mg}$ bid. The horizontal bar represents the mean of each data set. Panel B: The panel shows the 24-hour $\mathrm{sTXB}_{2}$ values (8pm) expressed as \% increase of the corresponding 12 hour (8am) value at day 7 post-surgery in the three randomized groups. Bars are geometric means and $95 \%$ confidence intervals. 
Figure 4 Serum $\mathbf{T X B}_{2}$ 12-to-24 hour recovery rates associated with different aspirin

regimens. The box-whisker plots represent median, IQR and outliers of recovery slopes of sTXB $_{2}$ measured between 8 am and $8 \mathrm{pm}$ on day 7 post-surgery in patients randomized to $100 \mathrm{mg}$ od, $200 \mathrm{mg}$ od or $100 \mathrm{mg}$ bid. Recovery slopes are expressed as ng/ml/hour of $\mathrm{sTXB}_{2}$.

\section{Figure 5. Pathophysiological mechanisms affecting aspirin pharmacodynamics after}

CPB surgery. Cardiac surgery and on-pump cardiopulmonary bypass in atherothrombotic disorders trigger platelet destruction and regeneration, acute inflammatory response, and in vivo platelet and endothelial activation. Platelet regeneration with accelerated COX1renewal over the 24-hour dosing interval impairs the pharmacodynamics of a conventional low-dose aspirin regimen, which is unable to restrain the enhanced platelet activation associated with cardiac surgery. The increased vascular biosynthesis of the antithrombotic prostanoid, $\mathrm{PGI}_{2}$, represents a homeostatic response to inflammation and platelet activation. 


$\begin{aligned} & \text { Aspirin } \\ & \text { intake }\end{aligned}$
witnessed

Figure 1 

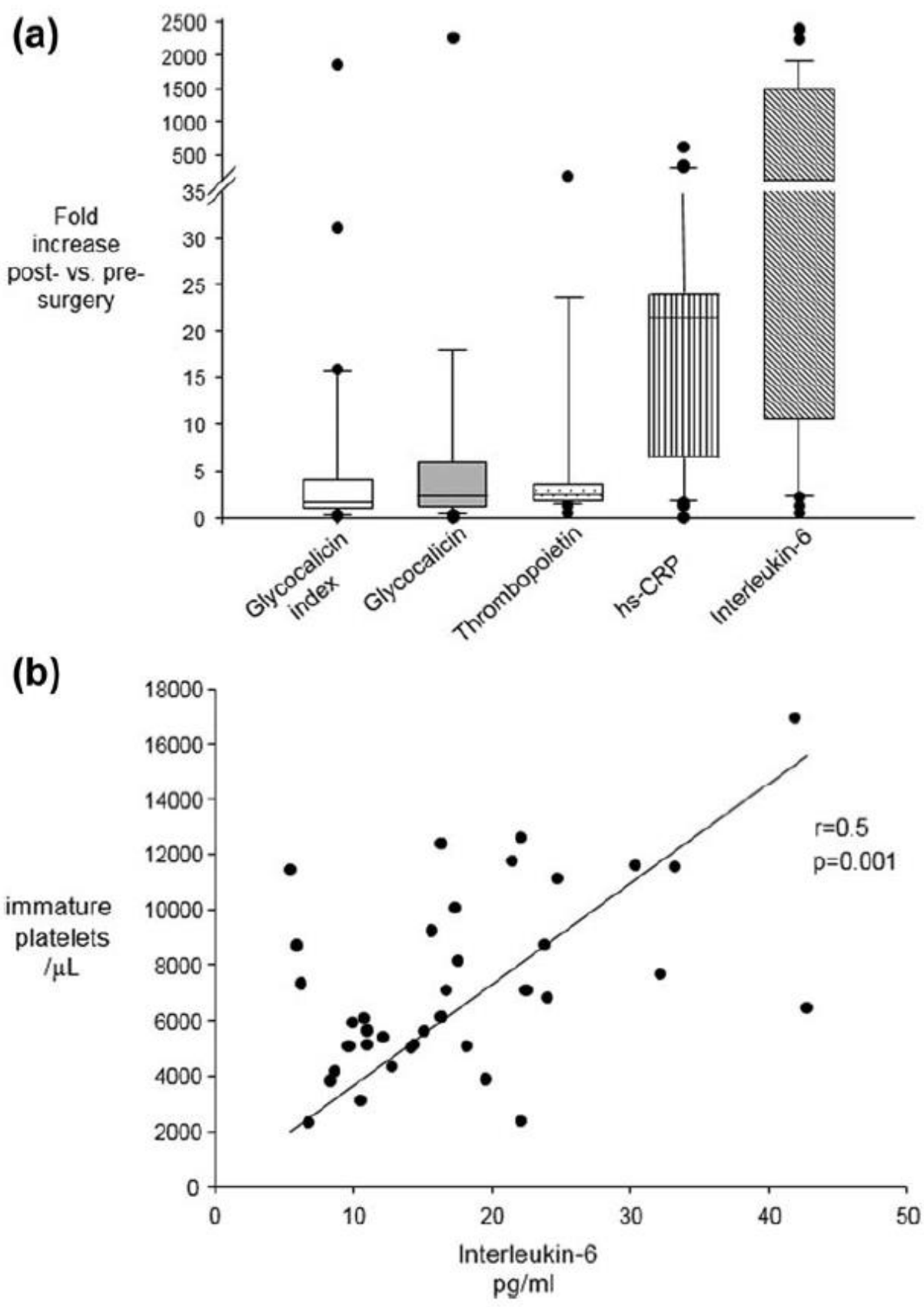

Figure 2 
(a)
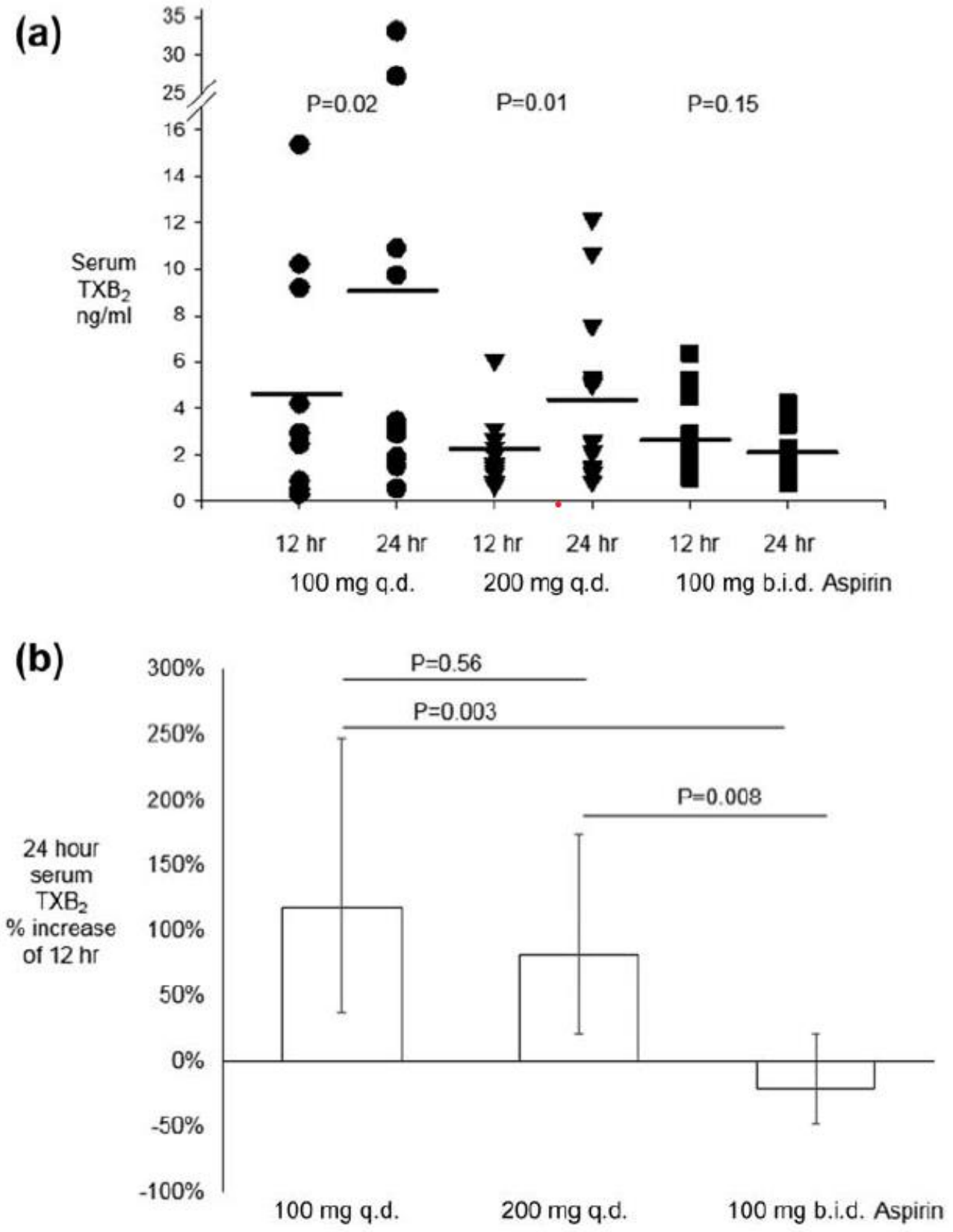

Figure 3 


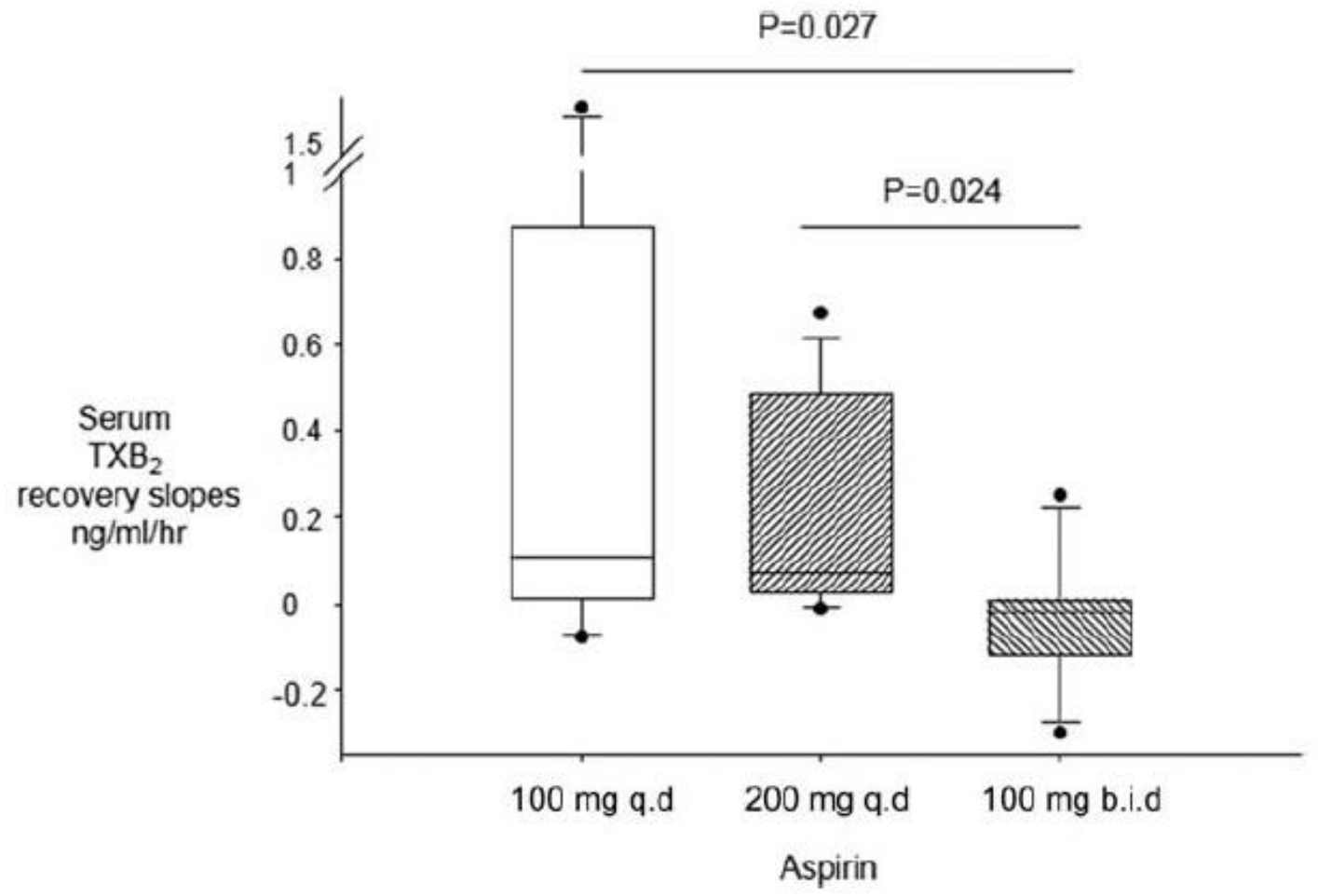

Figure 4 


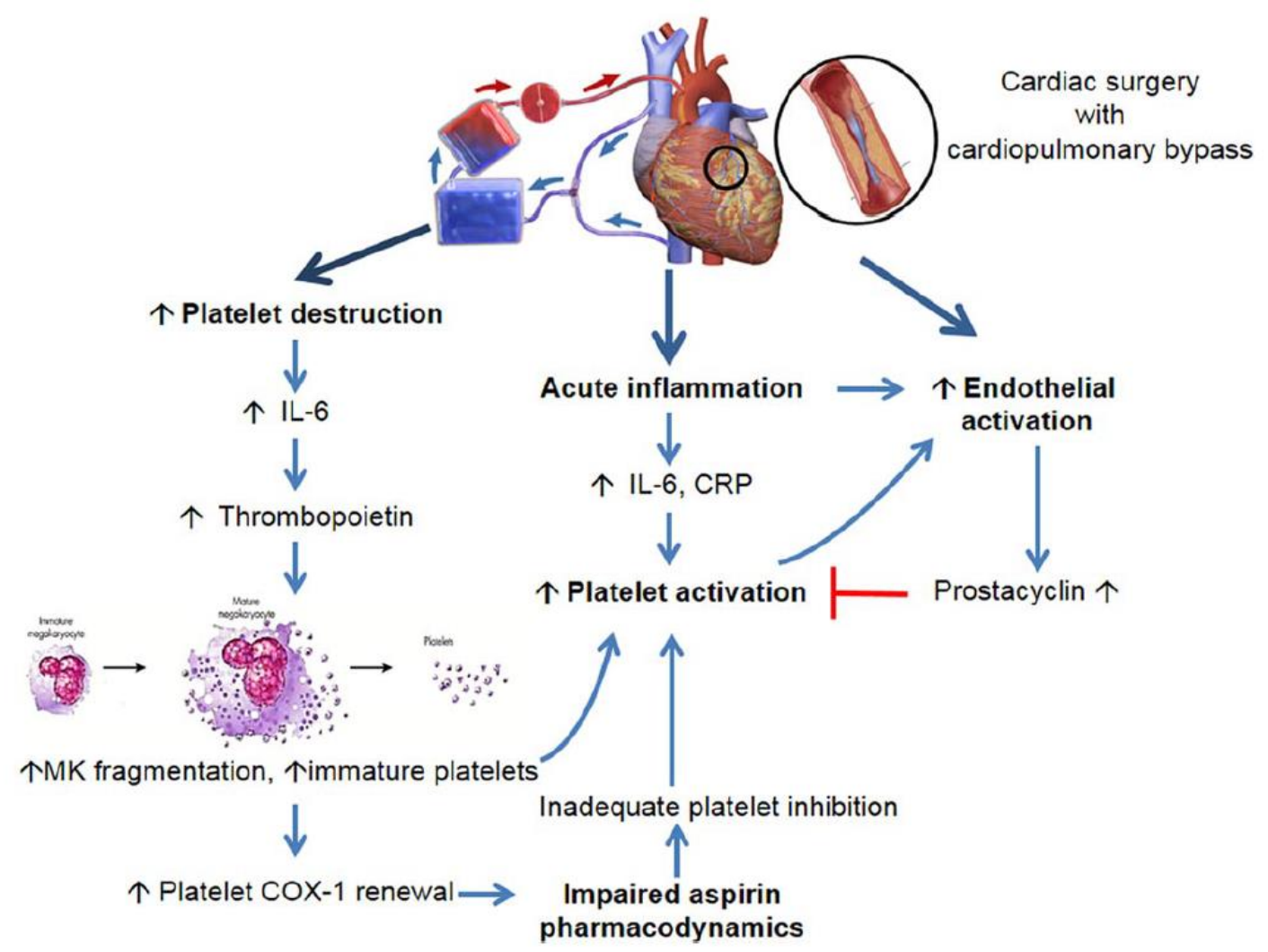

Figure 5 
Table 1. Urinary prostanoid metabolites and platelet counts at 8 am and 8 pm before and after cardiac surgery in 37 patients

\begin{tabular}{|c|c|c|}
\hline $\begin{array}{r}\text { Variable } \\
\text { Pre-surgery }\end{array}$ & $8 \mathrm{am}$ & $8 \mathrm{pm}$ \\
\hline $\begin{array}{l}\text { Urinary PGIM } \\
\text { pg/mg creatinine }\end{array}$ & $\begin{array}{c}133 \\
{[74-273]}\end{array}$ & $\begin{array}{c}150 \\
{[113-261]}\end{array}$ \\
\hline $\begin{array}{l}\text { Urinary TXM } \\
\text { pg/mg creatinine }\end{array}$ & $\begin{array}{c}132 \\
{[90-241]}\end{array}$ & $\begin{array}{c}102 \\
{[61-220]}\end{array}$ \\
\hline $\begin{array}{l}\text { Platelet count } \\
\times 10^{3} / \mu \mathrm{L}\end{array}$ & $\begin{array}{c}204 \\
{[176-239]}\end{array}$ & $\begin{array}{c}202 \\
{[183-232]}\end{array}$ \\
\hline $\begin{array}{l}\text { Immature platelet } \\
\text { count } \times 10^{3} / \mu \mathrm{L}\end{array}$ & $\begin{array}{c}5.64 \\
{[4.15-7.07]}\end{array}$ & $\begin{array}{c}6.12 \\
{[4.01-8]}\end{array}$ \\
\hline \multicolumn{3}{|l|}{ Post-surgery } \\
\hline $\begin{array}{l}\text { Urinary PGIM } \\
\text { pg/mg creatinine }\end{array}$ & $\begin{array}{c}268 \S \\
{[143-583]}\end{array}$ & $\begin{array}{c}249 \S \\
{[183-504]}\end{array}$ \\
\hline $\begin{array}{l}\text { Platelet count } \\
\times 10^{3} / \mu \mathrm{L}\end{array}$ & $\begin{array}{c}291 \S \\
{[246-425]}\end{array}$ & $\begin{array}{c}318 \S^{*} \\
{[272-433]}\end{array}$ \\
\hline $\begin{array}{l}\text { Immature platelet } \\
\text { count }\left(\times 10^{3} / \mu \mathrm{L}\right)\end{array}$ & $\begin{array}{c}6.44 \S \\
{[5.09-9.44]}\end{array}$ & $\begin{array}{c}7.53 \S * \\
{[5.68-11.01]}\end{array}$ \\
\hline
\end{tabular}

\begin{abstract}
Abbreviations: PGIM: $\mathrm{PGI}_{2}$ metabolite; TXM: TXA 2 metabolite; ${ }^{*} \mathrm{p}<0.01$ vs. 8 am value; $\S p<0.01$ vs. the corresponding pre-surgery value.
\end{abstract}


Table 2. Cytokine levels and hematological indexes measured at 8 am before surgery, at day 7 and 3 months after cardiac surgery in 37 patients

\begin{tabular}{|c|c|c|c|}
\hline Variable & $\begin{array}{l}\text { Before } \\
\text { surgery } \\
\text { (T1) }\end{array}$ & $\begin{array}{c}\text { Day } 7 \\
\text { after surgery } \\
\text { (T3) }\end{array}$ & $\begin{array}{c}\text { Month } 3 \\
\text { after surgery } \\
\text { (T5) }\end{array}$ \\
\hline Platelets $\times 10^{3} / \mu \mathrm{L}$ & $\begin{array}{c}204 \\
{[176-239]}\end{array}$ & $\begin{array}{c}291^{*} \\
{[246-424]}\end{array}$ & $\begin{array}{c}231 * \\
{[213-264]}\end{array}$ \\
\hline $\begin{array}{l}\text { Immature platelet count } \\
\left(\mathrm{x} 10^{3} / \mu \mathrm{L}\right)\end{array}$ & $\begin{array}{c}5.64 \\
{[4.150-7.074]}\end{array}$ & $\begin{array}{c}6.44 * \\
{[5.085-9.440]}\end{array}$ & $\begin{array}{c}5.64 \\
{[3.960-7.440]}\end{array}$ \\
\hline Platelet mass & $\begin{array}{c}0.21 \\
{[0.18-0.26]}\end{array}$ & $\begin{array}{c}0.3 * \\
{[0.26-0.42]}\end{array}$ & $\begin{array}{c}0.24 * \\
{[0.22-0.28]}\end{array}$ \\
\hline White blood cells $\times 10^{3} / \mu \mathrm{L}$ & $\begin{array}{c}6.6 \\
{[5.6-7.7]}\end{array}$ & $\begin{array}{c}9.2^{*} \\
{[8.2-11.2]}\end{array}$ & $\begin{array}{c}7.4^{*} \\
{[6-8]}\end{array}$ \\
\hline Granulocytes $\times 10^{3} / \mu \mathrm{L}$ & $\begin{array}{c}3.7 \\
{[3.2-4.8]}\end{array}$ & $\begin{array}{c}6.0 * \\
{[5.3-8]}\end{array}$ & $\begin{array}{c}4.2 \\
{[3.6-4.8]}\end{array}$ \\
\hline Monocytes $\times 10^{3} / \mu \mathrm{L}$ & $\begin{array}{c}0.4 \\
{[0.37-0.57]}\end{array}$ & $\begin{array}{c}0.8^{*} \\
{[0.6-0.9]}\end{array}$ & $\begin{array}{c}0.5 \\
{[0.4-0.6]}\end{array}$ \\
\hline Eosynophils $\times 10^{3} / \mu \mathrm{L}$ & $\begin{array}{c}0.15 \\
{[0.1-0.3]}\end{array}$ & $\begin{array}{c}0.3^{*} \\
{[0.2-0.4]}\end{array}$ & $\begin{array}{c}0.16 \\
{[0.1-0.3]}\end{array}$ \\
\hline Lymphocytes $\times 10^{3} / \mu \mathrm{L}$ & $\begin{array}{c}1.95 \\
{[1.5-2.3]}\end{array}$ & $\begin{array}{c}1.94 \\
{[1.3-2.3]}\end{array}$ & $\begin{array}{c}2.1 \\
{[1.7-2.6]}\end{array}$ \\
\hline Basophils $\times 10^{3} / \mu \mathrm{L}$ & $\begin{array}{c}0.03 \\
{[0.02-0.04]}\end{array}$ & $\begin{array}{c}0.03 \\
{[0.02-0.04]}\end{array}$ & $\begin{array}{c}0.03 \\
{[0.02-0.04]}\end{array}$ \\
\hline IL-6 pg/ml & $\begin{array}{c}0.31 \\
{[0.01-1.59]}\end{array}$ & $\begin{array}{c}16.3 * \\
{[10.7-22.3]}\end{array}$ & $\begin{array}{c}0.01 \\
{[0.01-1]}\end{array}$ \\
\hline hs-CRP mg/L & $\begin{array}{c}1.5 \\
{[0.7-6.4]}\end{array}$ & $\begin{array}{c}53 * \\
{[26-84]}\end{array}$ & $\begin{array}{c}3.6 \\
{[1.6-8]}\end{array}$ \\
\hline Thrombopoietin pg/ml & $\begin{array}{c}38 \\
{[22-62]}\end{array}$ & $\begin{array}{c}102 * \\
{[73-134]}\end{array}$ & $\begin{array}{c}86^{*} \\
{[77-104]}\end{array}$ \\
\hline Glycocalicin ng/ml & $\begin{array}{c}0.4 \\
{[0.1-0.9]}\end{array}$ & $\begin{array}{c}0.95 * \\
{[0.3-1.5]}\end{array}$ & $\begin{array}{c}0.71 * \\
{[0.4-1.2]}\end{array}$ \\
\hline Glycocalicin Index & $\begin{array}{c}0.5 \\
{[0.2-1]}\end{array}$ & $\begin{array}{c}0.68 * \\
{[0.2-1.3]}\end{array}$ & $\begin{array}{c}0.66 \\
{[0.4-1.2]}\end{array}$ \\
\hline
\end{tabular}

Abbreviations: IL: Interleukin; hs-CRP: high sensitivity C-reactive protein.

Values are medians and interquartile range. $* p<0.001$ vs. pre-surgery values 